\title{
Public Workshop Summary Report on Fiscal Year 2021 Generic Drug Regulatory Science Initiatives: Data Analysis and Model-Based Bioequivalence
}

Jieon Lee ${ }^{1}$, Yuqing Gong ${ }^{1}$, Sid Bhoopathy ${ }^{2}$, Charles E. DiLiberti ${ }^{3}$, Andrew C. Hooker ${ }^{4}$, Amin Rostami-Hodjegan ${ }^{5,6}$, Stephan Schmidt ${ }^{7}$, Sandra Suarez-Sharp ${ }^{8}$, Viera Lukacova ${ }^{8}$, Lanyan Fang ${ }^{1}$ and Liang Zhao ${ }^{1, *}$

On May 4, 2020, the US Food and Drug Administration (FDA) hosted an online public workshop titled "FY 2020 Generic Drug Regulatory Science Initiatives Public Workshop" to provide an overview of the status of the science and research priorities and to solicit input on the development of Generic Drug User Fee Amendments fiscal year 2021 priorities. This report summarizes the podium presentations and the outcome of discussions along with innovative ways to overcome challenges and significant opportunities related to model-based approaches in bioequivalence assessment for breakout session 4 titled, "Data analysis and model-based bioequivalence (BE)." This session focused on the application of model-based approaches in the generic drug development, with a vision of accelerating regulatory decision making for abbreviated new drug application assessments. The session included both podium presentations and panel discussions with three topics of interest: (i) in vitro study evaluation methods and their clinical relevance, (ii) challenges in model-based BE, (iii) emerging expertise and tools in implementing new BE approaches.

The availability of generic drug products in the market, specifically, complex generics, is limited by the lack of robust and sensitive measures to reliably and reproducibly demonstrate bioequivalence $(\mathrm{BE})$ between a proposed complex generic and its reference listed drug product. ${ }^{1}$ In recent years, the United States has seen increased adoption of generic products, with current estimates of $90 \%$ of filled prescriptions being a US Food and Drug Administration (FDA)-approved generic. ${ }^{2}$ Complex products generally consist of (i) products with complex active ingredients, formulations, routes of delivery, or dosage forms; (ii) complex drug-device combination products; and (iii) other products for which complexity or uncertainty concerning the approval pathway or possible alternative approach would benefit from early scientific engagement. Complex products, however, have historically faced less generic competition because of product-associated complexities, including their formulation, site of delivery, device, and patient-related aspects, all of which present challenges for generic development and approval. The means to demonstrate therapeutic equivalence of complex generic products in the past had relied on large, lengthy, and costly in vivo pharmacokinetic (PK) BE studies and comparative clinical end-point BE studies, which proved a significant logistical and financial burden to the healthcare system. ${ }^{3}$ The
FDA's Office of Generic Drugs (OGD) has generated and assessed model-integrated evidence to direct the design and evaluation of $\mathrm{BE}$ studies, enabling the assessment of alternative $\mathrm{BE}$ approaches for complex products. ${ }^{4-8}$ The OGD recognizes the need for stronger and more effective collaborations between stakeholders to address scientific gaps that present barriers to complex generic product development and/or abbreviated new drug application review. ${ }^{9}$ In this context, the OGD offers open forum discussions to improve transparency and predictability for generic drug applicants to help increase timely access to high-quality, lower cost generic drugs. To this end, on May 4, 2020, the FDA held the Financial Year 2020 Generic Drug Regulatory Science Initiatives Public Workshop to provide an overview of the status of the current fiscal year (FY) 2020 Generic Drug User Fee Amendments (GDUFA) science and research priorities, and to solicit input on the development of FY 2021 priorities. ${ }^{10}$ This report mainly summarizes the presentations and the associated discussions from the workshop's breakout session moderated by Dr Liang Zhao (FDA), titled, "Data analysis and model-based bioequivalence (BE)." The following three questions were discussed: (i) How to evaluate data from in vitro studies and which in vivo studies are clinically relevant (e.g., how to justify qualitative sameness (Q1)/quantitative

\footnotetext{
${ }^{1}$ Division of Quantitative Methods and Modeling, Office of Research and Standards, Office of Generic Drugs, Center for Drug Evaluation and Research, US Food and Drug Administration, Silver Spring, Maryland, USA; ${ }^{2}$ Absorption Systems, Exton, Pennsylvania, USA; ${ }^{3}$ Montclair Bioequivalence Services, LLC, Upper Montclair, New Jersey, USA; ${ }^{4}$ Department of Pharmacy, Uppsala University, Uppsala, Sweden; ${ }^{5}$ Centre for Applied Pharmacokinetic Research, University of Manchester, Manchester, UK; ${ }^{6}$ Certara, Princeton, New Jersey, USA; ${ }^{7}$ Center for Pharmacometrics \& Systems Pharmacology, Department of Pharmaceutics, University of Florida, Orlando, Florida, USA; ${ }^{8}$ Simulations Plus, Inc, Lancaster, California, USA. *Correspondence: Liang Zhao (Liang.Zhao@fda.hhs.gov)
}

Received September 22, 2020; accepted November 14, 2020. doi:10.1002/cpt.2120 
sameness (Q2)/physicochemical similarity (Q3) for equivalence assessment)? (ii) What are the challenges for industry in implementing modeling and simulation methods to support more efficient regulatory BE pathways? (iii) What are the emerging expertise/tools in implementing new $\mathrm{BE}$ approaches?

These topics are closely related to the FY 2020 GDUFA Research Science Priorities, "Tools and methodologies for BE and therapeutics equivalence evaluation," which include (i) improvement of quantitative pharmacology and BE trial simulation for complex generic drug products; (ii) integration of predictive dissolution, physiologically-based pharmacokinetic (PBPK) and pharmacokinetics (PK) / pharmacodynamics (PD) models for BE standards establishment; (iii) recognition of the role of excipients to expand biowaivers of the Biopharmaceutics Classification System (BCS) of class 3 drug products; and (iv) development of methods and integrated technological solutions to leverage large datasets. The expert discussant panel include: Liang Zhao, Amin Rostami, Andrew Hooker, Charlie DiLiberti, Glenys Barber, Sandra Suarez-Sharp, Viera Lukacova, Stella C. Grosser, Stephan Schmidt, Sid Bhoopathy, Raja Velagapudi, and Lanyan Fang.

\section{MEETING SUMMARY}

\section{Evaluation methods and clinical relevance of data from in vitro studies}

Scientific presentations. The first presentation, given by $\mathrm{Mr}$ Charles E. DiLiberti (Montclair Bioequivalence Services, LLC, Montclair, New Jersey), focused on the need of using modeling approaches to support relaxation of compositional/ microstructural criteria to qualify for streamlined $\mathrm{BE}$ approaches. Currently, the FDA's OGD allows for streamlined $\mathrm{BE}$ approaches if certain narrow compositional/microstructural criteria are met. For example, BCS class 3 biowaivers ${ }^{11}$ can be used to avoid the need to perform in vivo BE studies, but require that the proposed generic and reference product formulations meet specific compositional similarity requirements. Similarly, "in vitro only" BE pathways for complex and locally acting drug products ${ }^{12-15}$ avoid the need to conduct expensive in vivo $\mathrm{BE}$ studies but require that the generic and reference products are qualitatively $(\mathrm{Q} 1)$, and quantitatively $(\mathrm{Q} 2)$ the same, and microstructurally (Q3) similar. Mr DiLiberti pointed out that meeting these narrow $\mathrm{Q} 1 / \mathrm{Q} 2 / \mathrm{Q} 3$ standards often requires exhaustive reverse engineering of the reference product using state-of-the-art analytical techniques. Matching microstructurally (Q3) can necessitate process optimization via hundreds of trial formulations. Patents sometimes block the ability to use a Q1/Q2/Q3 formulation, even if the sponsor can do so from a technical standpoint.

Mr DiLiberti further elaborated that Q1/Q2/Q3 requirements can sometimes be so onerous that generic sponsors forego the in vitro BE pathway and opt for the human BE study pathway instead, which defeats the purpose of the streamlined pathway. Under extreme situations, the generic product is sometimes abandoned altogether because the costs of the "in vitro only" and human BE pathways are both too high. Therefore, he argued, there is a significant need to develop modeling approaches to justify relaxation of the narrow and probably overly conservative compositional and microstructural requirements currently in place to qualify for "in vitro only" BE pathways.

Secondly, Dr Sid Bhoopathy (Absorption Systems, Exton, Pennsylvania) presented potential areas that can be focused on expanding the reach and utility of model-informed BE. The potential areas include (i) designing the Q1/Q2/Q3 safe space; i.e., determination of the relative space within which the critical quality attributes (CQAs) can be varied without impacting the in vivo outcome; (ii) strengthening the regulatory applicability of PBPK with product category specific input parameters; (iii) complementing model-based BE with other modeling approaches such as PK/PD; and (iv) widening the utility of this methodology to BCS class 3 biowaivers and complex ophthalmic products. The quality and relevance of input (in vitro/ nonclinical) parameters are critical for improved models and recommendations were made on best practices for generating such data.

Two case studies were presented on the expansion potential of model-informed BE by Dr Bhoopathy. The first case study was on the use of novel in vitro input parameters from a combined dissolution and absorption system to justify wider tolerance of excipient differences for BCS class 3 biowaivers, ${ }^{16}$ which are currently applied during the investigational new drugs / new drug applications or abbreviated new drug applications. The work, which is currently ongoing, is divided into two phases. Phase 1 is the evaluation of the effect of 15 commonly used excipients, at varying and clinically relevant concentrations, on the permeation of a cassette of five model drugs using the combined dissolution and absorption apparatus. Four of these model drugs are BCS class 3 and one of them is BCS class 1 . The next phase of this project will involve testing the individual BCS Class 3 drug products with published clinical data using different formulations, in the in vitro model to understand the correlation between generated in vitro data and available clinical data. This work can lead into investigating in-vitro-in-vivo correlation (IVIVC) using PBPK models to set clinically relevant space (also known as safe space) in terms of Q2 differences for these products, setting-up excipient exception categories for class 3 biowaivers, and providing alternative pathways for data evaluation when justifying the lack of impact of excipient change on class 3 product BE. The second case study was on the use of modeling to determine $\mathrm{BE}$ for a chronic complex ophthalmic product. Considerations were discussed for evaluating the formulation "bio-morphology" (change in the formulation's properties when interacting with the bio-membrane / site of action) and how to characterize its impact on BE. Formulation biopharmaceutical properties relevant to an ophthalmic suspension include, e.g., its residence time upon administration, transcorneal permeation, alternative permeation pathways on the surface of the eye that may impact its aqueous humor availability, etc. It is important to understand how the physicochemical properties of the formulation relate to these biopharmaceutical aspects, and the tools/models available to assist in the identification of these interactions, and thereby the formulation CQAs. The subsequent challenges in establishing IVIVC for identified ophthalmic product CQAs due to the lack of human in vivo data were also shared. Additional research is needed 
on using surrogate measures for ophthalmic product IVIVC and model validation.

Summary of the discussion. Drs Sandra Suarez-Sharp and Viera Lukacova (Simulations Plus Inc., Lancaster, California) shared their opinions on approaches to justify Q1/Q2/Q3 deviation for $\mathrm{BE}$ assessment. For example, for solid dosage forms, specifically for the oral route, in vitro release/dissolution (when adequately developed) is the only in vitro quality attribute which provides insight on the rate and extent of in vivo absorption from formulation performance perspective. As such, it may be implemented as a stand-alone test or critical input into physiologically-based biopharmaceutics modeling (PBBM)/ PBPK models for demonstration of BE for complex generic drug products. $^{17,18}$

One feasible approach is through the implementation of Quality by Design, where bio-predictive dissolution methods could be used as an end point in design of experiments, allowing for the selection of critical formulation and manufacturing variables (CFMV), namely Q1, Q2, and Q3. The clinical relevance of these critical variables and their ranges can then be assessed once an in vitroin vivo link and safe space have been established via, e.g., PBBM/ PBPK modeling (Figure 1). These steps are essential to ensure consistent performance compared with the biobatch and allow for flexibility/deviation on these critical quality parameters. One key element that should be considered in the creation of this link between CFMV, dissolution, and in vivo performance is the in vivo evaluation of variables representing the extreme interactions among them, facilitating the identification of a rank order relationship. The evaluation of one variable by itself, ignoring the potential interaction among the CFMV, creates uncertainty in the applicability of PBBM/PBPK models used to facilitate the establishment of the desired in vitro-in vivo link.

The rest of the panel discussion involved debate among the meeting participants regarding a few points. Firstly, participants made comments on the cost of generic drug product development. Participants suggested expanding the use of modeling and simulations for drug products of which the reference listed drug is from outside the United States with known Q1/Q2/Q3, and to reduce the cost of conducting clinical BE studies. Secondly, a question was raised regarding the need for Q1/Q2 sameness of locally acting oral liquid products. Dr Bhoopathy used BCS class 3 drugs as an example to answer this question. Since BCS class 3 drugs are highly soluble and rapidly dissolving, the $\mathrm{Q} 1 / \mathrm{Q} 2$ requirement delineated for BCS class 3 drugs should also be applied to oral liquid products. The expanded excipient range can be applied, and the model-based approach can be used based on in vitro experimental results to provide an informed decision on oral liquid products. Several suggestions were made from FDA participants, including possibilities of sharing PBPK models or posting reviews in a public domain and constructing a database indicating excipients that do not affect $\mathrm{BE}$ to reduce the time and cost of generic drug developments.

\section{Challenges in implementing modeling and simulation to support more efficient regulatory BE pathways}

Scientific presentations. Dr Amin Rostami-Hodjegan (University of Manchester, Manchester, UK) presented virtual bioequivalence requirements for prudent use of PBPK in uncharted territories. The related information can be found in his published articles

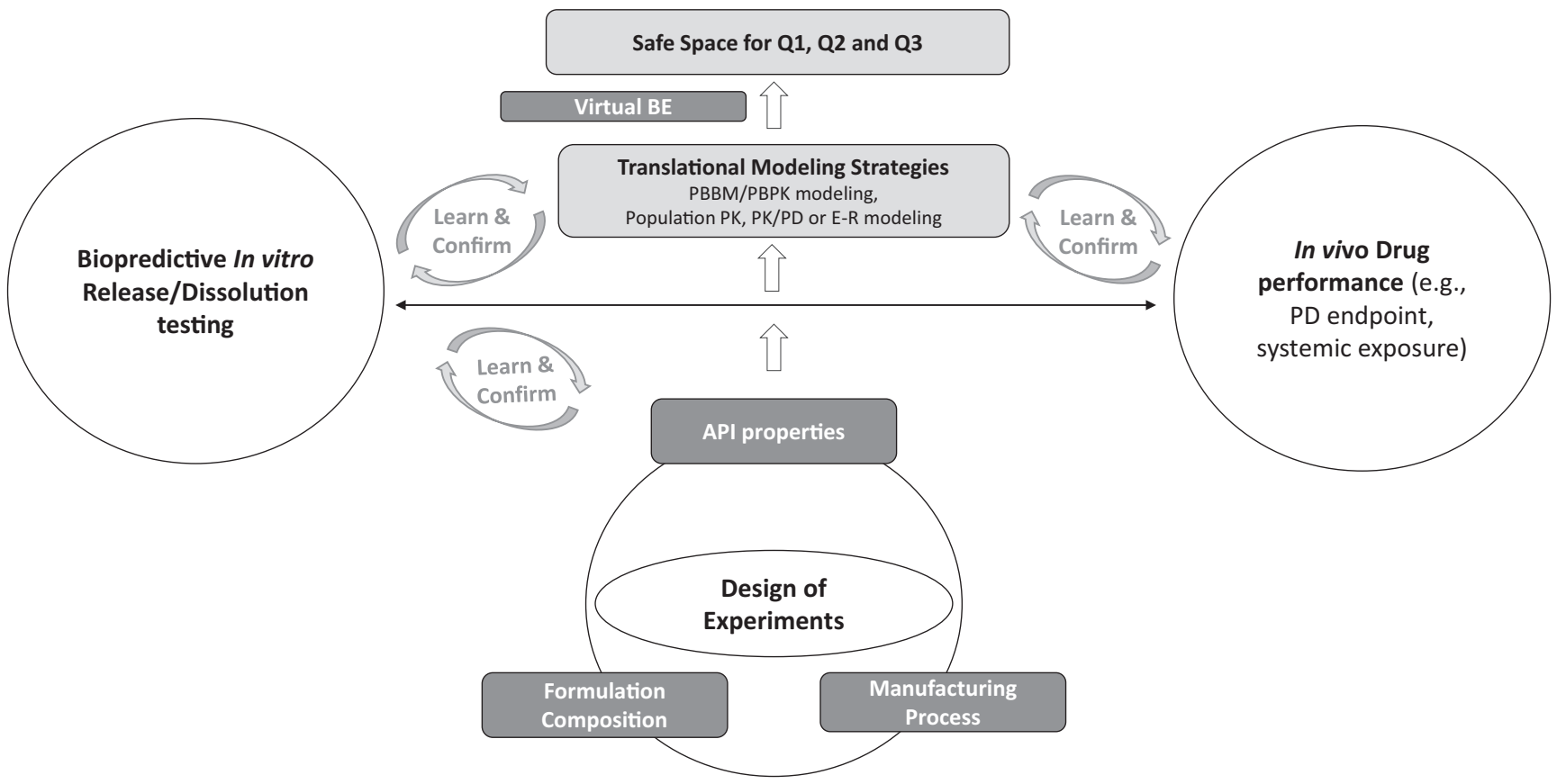

Figure $1 \mathrm{~A}$ proposed in vitro-in vivo link to assess the clinical relevance of critical variables and to establish the safe space for Q1/Q2/Q3. API, active pharmaceutical ingredient; BE, bioequivalence; E-R, exposure-response; PBBM, physiologically-based biopharmaceutics modeling; PBPK, physiologically-based pharmacokinetic; PD, pharmacodynamic; PK, pharmacokinetic; Q1, qualitative sameness; Q2, quantitative sameness; Q3, physicochemical similarity. 
regarding the evaluation of PBPK prediction success. ${ }^{19,20}$ "Model-informed drug development" (MIDD) has now become synonymous with the "integration of all relevant and available information" in helping with decision making during the process of drug development. ${ }^{21}$ Despite the formal recognition of MIDD in Section 3 of the Prescription Drug User Fee Act by the FDA, there are many pharmaceutical companies where MIDD is not common practice or is not even known as a path for drug development. Similar issues surround the applications of MIDD for GDUFA. This could be a bigger problem during generic drug development as it involves many smaller companies, who are at a disadvantageous position when it comes to internal bandwidth to support all the quantitative integration of modeling efforts needed. Availability of ready-made and user-friendly tools plays a big role in moving MIDD from a "luxurious nice-to-have" tool to an "essential must-have" kit.

PBPK models are a subsector within the broader discipline of mechanistic biological systems modeling. PBPK models incorporate mechanistic understanding of absorption and drug disposition and address the "interplay" between drug, formulation, and attributes of physiology and biology in each set of patients (target population). The modern use of PBPK (in the $21^{\text {st }}$ century) has been less focused on the models but more on "systems" information (in various conditions) which are independent of any drug and formulation. The interaction of these characteristics with those of the drug and formulation determines the impact on specific PK outcomes.

The specific system parameters and modeling issues are associated with better virtual bioequivalence. Since the major focus of PBPK so far has been the disposition of the new drug entities, system parameters related to absorption of the drugs (that concerns generic drugs) in the gastrointestinal system and other routes of entry are not well defined. Better tools for modeling the in vitro studies rather than using summary information and point measures is essential for the ability to incorporate valid translational steps in going from in vitro data to in vivo consequences. Dr RostamiHodjegan stated that regulatory requirements for model-derived values will play a big role in making these popular as an alternative to less-informative "point-measures." In addition, interoccasion variability (IOV) is rarely known for physiological parameters concerning various routes of administration. However, the BE studies with various classes of drugs where the replicate studies are used may help to construct the best estimates for IOV of physiological values for a wide range of drugs and formulations. Dr RostamiHodjegan suggested that the internal database with the OGD could potentially be used for such an exercise.

Summary of discussion. The panel discussion focused on the challenges of using modeling and simulation to support efficient regulatory BE pathways, especially PBPK models. Dr Lukacova agreed with the speaker that a good tool for the mechanistic simulations for the in vitro system is needed. She commented that a tool to evaluate the systemic parameters and to estimate the IOV is necessary. Dr Lukacova pointed out the research needs to evaluate the effects of the excipients on the physiologic system, such as the effect on permeability, transporters and enzymes, transit time, $\mathrm{pH}$, and food effect. Dr Zhao (FDA) agreed with the comment and pointed out that a well-established in vitro modeling could help determine the food effect which potentially helps to determine the waiver of doing only a fasting BE study. ${ }^{17,22}$ There was an additional question being raised from participants regarding the method to identify the potential complex correlations between all the possible systemic parameters. Dr Rostami-Hodjegan stated that this could be done by using well-known mathematical approaches such as the Cholesky decomposition of large matrix of intercorrelations and the subsequent Monte Carlo sampling for simulating any systems with multiple correlated variables. ${ }^{23}$ However, apart from proteomics, where we have generated the complex correlations between proteins, transporters, and enzymes, most of the physiological correlations are only one-to-one relation and some of them are still under development.

\section{Emerging expertise and tools in implementing new BE approaches}

Scientific presentations. Firstly, Dr Andrew Hooker (Uppsala University, Uppsala, Sweden) presented methods for improved $\mathrm{BE}$ assessment through the use of population pharmacometric (nonlinear mixed-effect) models. Two different uses of these pharmacometric models were presented. The first one was a model-informed approach where pharmacometric models are used to understand and optimize the operating characteristics of standard BE methods and designs. For example, using simulations from a well-defined pharmacometric model, fixed covariate effects could be utilized in a standard analysis of a parallel group study to reduce the residual variability and increase the power of the analysis. A second example demonstrated how pharmacometric model simulations could guide the use of switch studies to replace unreasonably long crossover steady-state studies for long-acting injectable products. In this example, the simulations allowed for an adjustment of the standard BE limits as well as an assessment of the needed sample size in these shorter studies. A third example demonstrated the use of model simulations to understand when steady state would be achieved in a study and gave an example where standard methods of assessing steady state would be wildly inaccurate. The second approach proposed for the use of pharmacometric models was through the direct application of these models in the assessment of $\mathrm{BE}$ (model-integrated approaches). In these approaches, a model or a cohort of models are fit to BE data. From the resulting model parameter estimates and uncertainty, population simulations can be used to assess BE. The described model-integrated BE analysis method has been shown to have acceptable type I error and higher power than standard noncompartmental analysis-based methods in sparse sampling scenarios, and could also be useful in BE assessment for highly variable drugs. ${ }^{24}$ The model-informed and model-integrated strategies have the potential to reduce study duration and/or sample size, leading to more feasible BE studies in a number of situations.

Secondly, Dr Stephan Schmidt (University of Florida, Orlando, Florida) presented a model and systems-based approach to address efficacy and safety questions related to generic substitution. 
Approved drug products are considered to be therapeutic equivalents if they are pharmaceutical equivalents for which $\mathrm{BE}$ has been demonstrated, and they can be expected to have the same clinical effect and safety profile when administered to patients under the conditions specified in the labeling. Dr Schmidt noted that the conventional BE limit (i.e., 90\% confidence interval within 80-125\%) may not be stringent enough (such as narrow therapeutic index drugs) or too stringent depending on the steepness of the exposure-response profiles for efficacy and safety as well as the associated therapeutic window for a given drug product. The use of combined modeling and simulation approaches integrating drug-specific, formulation-specific, and patient-specific properties into an overarching framework provides a unique opportunity to leverage available information in a strictly quantitative fashion to assess $\mathrm{BE}$ and to refine $\mathrm{BE}$ standards, particularly in circumstances (e.g., complex generics) where head-to-head clinical end-point trials are time and cost prohibitive. Close collaboration at the FDA throughout the product life cycle may be desirable because (i) exposure-response relationships relevant for therapeutic equivalence are typically available from new drug applications and can be used to identify BE standards and to develop product-specific guidance; (ii) BE standards and evaluation procedures also apply to new drug development when scaling between development and to-be-marketed formations; and (iii) potential BE issues may be anticipated prior to the brand name product coming off patent. Future research should be conducted to gain a better understanding of the role of excipients on dissolution and permeability as well as variability therein.

Summary of the discussion. The panel discussion mainly focused on the availabilities of modeling tools. Even though various modeling tools are available, it was noted that modeling experts are limited to apply those tools. Dr Rostami-Hodjegan discussed the difference between "open science" and "open source" approaches. It was noted that an "open science" approach has been used widely among researchers because of the clarity in user guidance and the quality and transparency of the information provided. Participants suggested that using a model search base would help avoid potential bias of the model selection. In addition, Dr Hooker suggested that while open science approaches are very welcome (and perhaps more economically viable), open source approaches are even more forthcoming with what is being done and how it is being done.

\section{CONCLUSION}

Dr Lanyan Fang (FDA) gave a concluding remark on the session. The session highlighted the current and emerging model-based approaches in BE assessment. Several take-home messages can be listed that summarized the presentations and discussions: (i) Opportunities exist to apply modeling methods in BE assessment, with a vision of accelerating generic drug development and regulatory assessment of generic products by modeling and simulation; (ii) The modeling methods have been used to probe the impact of deviations from narrow compositional/microstructural criteria on expected product performance and in vivo BCS 3 waiver expansion for other "nonqualifying" oral drug product; (iii) For solid oral dosage forms, in vitro release/dissolution testing is the quality attribute that best represents the drug product performance; and (iv) Expanding the reach and utility of modeling-supported BE to more product categories.

The challenges for the industry in implementing PBPK/absorption models to support more efficient $\mathrm{BE}$ methods include, but not limited to (i) uncertainty of the model which best describes the system; (ii) bias or mis-specification of model parameters; and (iii) understanding of the biological systems. There are emerging quantitative methods and modeling expertise/tools (e.g., model-informed and model-based $\mathrm{BE}$ approaches such as virtual $\mathrm{BE}$ simulation, PBPK modeling, combined bioinformatics, etc., in implementing new $\mathrm{BE}$ approaches for various purposes).

The session also facilitated the discussion on the knowledge gaps and challenges related to the use of model-based and model-integrated approaches. More collaborations among the FDA, industry, and academia are needed to close scientific gaps in the use of modeling and simulation in generic drug development and to reach global harmonization.

\section{FUNDING}

S.B.'s work was supported by US Food and Drug Administration (FDA) contract 75F40119C10127. S.S.'s work was supported by FDA grant U01 RFA-FD-14-011. A.C.H.'s work was supported by FDA grant HHSF223201710015C. The others' work reported in this article are not funded/sponsored.

\section{CONFLICTS OF INTEREST}

The opinions expressed in this article are those of the authors and do not necessarily represent the views or policies of their affiliated organizations/agencies. A.R.-H. would like to declare that he is the Director of Centre for Applied Pharmacokinetics Research (CAPKR). The activities of CAPKR are sponsored by a consortium of pharmaceutical companies (including Merck, GSK, Eli Lily, Genentech, J\&J, AbbVie, EMD Serono, Certara, and Takeda) in addition to research grants from government and research councils. As the Chief Scientific Officer and Senior Vice President of Research and Development at Certara, A.R.-H. has been involved in overseeing the development of software tools which are used by a large group of pharmaceutical companies during drug discovery and development, particularly in the area of physiologicallybased pharmacokinetics and quantitative systems pharmacology. A.C.H. is a Professor of Pharmacometrics at Uppsala University; some of his research has been funded by pharmaceutical companies. A.C.H. is also an advisor to the pharmaceutical consulting company Pharmetheus $A B$. All other authors declared no conflicts of interest.

\section{ACKNOWLEDGMENTS}

The authors gratefully acknowledge Drs. Glenys Barbers, Stella C. Grosser, Raja Velagapudi for insightful comments and discussion.

\section{DISCLAIMER}

The opinions expressed in this manuscript are those of the authors and should not be interpreted as the position of the US Food and Drug Administration.

(C) 2020 The Authors Clinical Pharmacology \& Therapeutics (C) 2020 American Society for Clinical Pharmacology and Therapeutics

1. US Food and Drug Administration. GDUFA reauthorization performance goals and program enhancements fiscal years 2018-2022 <https://www.fda.gov/media/101052/download> (2016).

2. The Association for Accessible Medicines. The case for competition: 2019 generic drug \& biosimilars access \& savings in 
the U.S. report <https://accessiblemeds.org/sites/default/files/ 2019-09/AAM-2019-Generic-Biosimilars-Access-and-Savings-USReport-WEB.pdf> (2019).

3. US Food and Drug Administration. Food and Drugs, 21 USC §314.3. <https://www.accessdata.fda.gov/scripts/cdrh/cfdocs/ cfcfr/CFRSearch.cfm?fr=314.3> (2019).

4. Zhao, L., Kim, M.-J., Zhang, L. \& Lionberger, R. Generating model integrated evidence for generic drug development and assessment. Clin. Pharmacol. Ther. 105, 338-349 (2019).

5. Lionberger, R. Decision science for generic drug development and review. J. Clin. Pharmacol. 59, 1249-1251 (2019).

6. Kim, S. et al. Evaluating the clinical impact of formulation variability: a metoprolol extended-release case study. J. Clin. Pharmacol. 59, 1266-1274 (2019).

7. Brown, J.D., Henriksen, C., Vozmediano, V. \& Schmidt, S. Realworld data approaches for early detection of potential safety and effectiveness signals for generic substitution: a metoprolol extended-release case study. J. Clin. Pharmacol. 59, 1275-1284 (2019).

8. Basu, S. et al. Physiologically based pharmacokinetic modeling to evaluate formulation factors influencing bioequivalence of metoprolol extended-release products. J. Clin. Pharmacol. 59, 1252-1263 (2019).

9. Lesko, L.J., Fang, L., Schmidt, S. \& Trame, M.N. An integrated bioinformatics and quantitative modeling approach to investigate potential claims of oral generic drug product bioinequivalence: introduction. J. Clin. Pharmacol. 59, 1245-1248 (2019).

10. US Food and Drug Administration. FY 2020 generic drug regulatory science initiatives public workshop <https://www.fda. gov/news-events/fda-meetings-conferences-and-workshops/ fy-2020-generic-drug-regulatory-science-initiatives-public-works hop-05042020-05042020> (May 4, 2020).

11. US Food and Drug Administration. Guidance for industry: waiver of in vivo bioavailability and bioequivalence studies for immediaterelease solid oral dosage forms based on a biopharmaceutics classification system <https://www.fda.gov/media/70963/ download> (2017).

12. US Food and Drug Administration. Draft guidance on acyclovir <https://www.accessdata.fda.gov/drugsatfda_docs/psg/ Acyclovir_topical\%20cream_RLD\%2021478_RV12-16.pdf> (2016).

13. US Food and Drug Administration. Draft guidance on silver sulfadiazine cream <https://www.accessdata.fda.gov/ drugsatfda_docs/psg/Silver\%20sulfadiazine_topical\%20cream NDA\%20017381_RC05-17.pdf> (2017).
14. US Food and Drug Administartion. Draft guidance on perflutren <https://www.accessdata.fda.gov/drugsatfda_docs/psg/Perfl utren\%20Liposomal\%2OInjection\%2ORLD\%20021064\%20RC\%20 09-2018\%20.pdf> (2018).

15. US Food and Drug Administration. Draft guidance on triamcinolone acetonide <https://www.accessdata.fda.gov/drugs atfda_docs/psg/Triamcinolone\%20acetonide_intravitreal\%20inj ectable_NDA\%20022048_RC07-18.pdf> (2018).

16. Chen, M.-L. et al. Summary report of second EUFEPS/AAPS conference on global harmonization in bioequivalence. Eur. J. Pharm. Sci. 127, 24-28 (2019).

17. Heimbach, T. et al. Dissolution and translational modeling strategies toward establishing an in vitro-in vivo link - a workshop summary report. AAPS J. 21, 29 (2019).

18. Pepin, X.J.H. et al. Current state and future expectations of translational modeling strategies to support drug product development, manufacturing changes and controls: a workshop summary report. J. Pharm. Sci. (2020). https://doi.org/10.1016/j. xphs.2020.04.021.

19. Margolskee, A. et al. IMI - Oral biopharmaceutics tools project -Evaluation of bottom-up PBPK prediction success part 2: an introduction to the simulation exercise and overview of results. Eur. J. Pharm. Sci. 96, 610-625 (2017).

20. Chen, Y. et al. Physiologically-based pharmacokinetic modelinformed drug development for fenebrutinib: understanding complex drug-drug interactions. CPT Pharmacometrics Syst. Pharmacol. 9, 332-341 (2020).

21. European Medicines Agency. Guideline on the pharmacokinetic and clinical evaluation of modified release dosage forms (EMA/ CPMP/EWP/280/96 Corr1) <https://www.ema.europa.eu/en/ documents/scientific-guideline/guideline-pharmacokinetic-clini cal-evaluation-modified-release-dosage-forms_en.pdf> (2014).

22. Zhang, X. et al. Mechanistic oral absorption modeling and simulation for formulation development and bioequivalence evaluation: report of an FDA public workshop. CPT Pharmacometrics Syst. Pharmacol. 6, 492-495 (2017).

23. Liu, D., Li, L., Rostami-Hodjegan, A., Bois, F.Y. \& Jamei, M. Considerations and caveats when applying global sensitivity analysis methods to physiologically based pharmacokinetic models. AAPS J. 22, 93 (2020).

24. Hooker, A.C.N.H., Karlsson, M.O. \& Chen, X. Development and comparison of model-based bioequivalence analysis methods on sparse data. Paper presented at the 10th American Conference on Pharmacometrics (ACOP10), Orlando, Florida, October 20-23, 2019. 